Check for updates

Cite this: RSC Adv., 2018, 8, 41872

\title{
Investigation of the dynamic changes in the chemical constituents of Chinese "Laba" garlic during traditional processing $\dagger$
}

\author{
Jian Liu, ${ }^{\text {ab }}$ Wei Guo, ${ }^{\mathrm{b}}$ Minli Yang, ${ }^{\mathrm{b}}$ Lixia Liu, ${ }^{\mathrm{b}}$ Shengxiong Huang, ${ }^{\mathrm{a}}$ Liang Tao, ${ }^{\mathrm{c}}$ \\ Feng Zhang ${ }^{*}{ }^{* b}$ and Yongsheng Liu*a
}

"Laba" garlic is a famous traditional garlic product, particularly popular with the people in Northern China. The processing time plays an important role on the chemical constituents of "Laba" garlic. Here, we investigated the composition of "Laba" garlic during traditional processing using a non-targeted metabolomics approach. Through using gas chromatography mass spectrometry (GC-MS) and liquid chromatography mass spectrometry (HPLC-MS/MS) with multivariate analysis, a total of 20 volatile metabolites, 16 primary metabolites and 15 phenolic compounds were identified as notable changed compounds due to the traditional processing $(p<0.05)$. The characteristic flavor of "Laba" garlic was formed mainly by the decreased content of organosulfur compounds and the increased content of nonorganosulfur compounds. In addition, this study also proposed the metabolic pathway of primary metabolites and phenolic compounds in garlic samples during processing. Most primary metabolites including lactic acid, isocitric acid, L-leucine, L-proline, D-fructose, D-glucose and erythritol increased from day 3, and reached the maximum level at day 12, which were thought to be the foundation for the sweet and sour taste of "Laba" garlic. Although the concentration of the 15 phenolic compounds from day 3 to day 42 was notably higher than the raw garlic ( 0 day), the antioxidant activities showed a decreasing trend from day 0 to day 28. The correlation analysis result revealed that notable positive associations were presented between organosulfur compounds, organic acids, amino acids and antioxidant activities. Furthermore, day 12 was found to be the most suitable time to obtain the "Laba" garlic considering its colour, flavor, taste and physiological function. These results are helpful in comprehending metabolism changes and physiological function of "Laba" garlic traditional processing.

\author{
Received 23rd November 2018 \\ Accepted 27th November 2018 \\ DOI: $10.1039 / \mathrm{c} 8 \mathrm{ra09657k}$ \\ rsc.li/rsc-advances
}

\section{Introduction}

Garlic (Allium sativum L.) is an important vegetable and has been widely consumed throughout the world for centuries, particularly popular in Europe, Asia and Africa., ${ }^{\mathbf{1 , 2}}$ Garlic is well known not only for its abundant nutrients but also for potential health benefits such as antibacterial, antioxidative and antiviral effects. $^{3-5}$ Currently, garlic is processed in diverse forms like garlic powder, garlic oil, garlic puree and black garlic, in order to satisfy the needs of consumers and the markets. ${ }^{6,7}$

Among the numerous garlic products, a famous homemade Chinese food called "Laba" garlic is very much enjoyed by

${ }^{a}$ School of Food Science and Engineering, Hefei University of Technology, Hefei 230009, China

${ }^{b}$ Institute of Food Safety, Chinese Academy of Inspection \& Quarantine, Beijing 100176, China. E-mail: fengzhang@126.com; fengzhangchem@yahoo.com; Fax: +8610-53898008; Tel: +86 13651290763

${ }^{c}$ College of Food Science and Technology, Yunnan Agricultural University, Kunming 650201, China

$\uparrow$ Electronic supplementary information (ESI) available. See DOI: 10.1039/c8ra09657k people in Northern China. "Laba" in Chinese represents the eighth day of the twelfth lunar month, and "Laba" garlic is traditionally consumed as a condiment or a vegetable in China. The green colour is one of the prominent features of "Laba" garlic compared with other garlic products. And previous research studies on "Laba" garlic have focused on its greening mechanism in the past decades. ${ }^{9-11}$ On the occurrence of breaking down the garlic cell membrane by vinegar, alliin and alliinase in garlic react rapidly and further form allicin. Based on allicin and through multistep enzymatic and non-enzymatic reactions, the yellow and blue pigments of "Laba" garlic are generated. ${ }^{12}$ Thus, it can be inferred that numerous compounds might be changed in "Laba" garlic during its traditional processing, including carbonyl compounds and amino acids. ${ }^{13-15}$

The traditional homemade processing of Chinese "Laba" garlic is through packing aged garlic into jars and then covering with vinegar for several days (more than a week) at low temperatures, which is very simple and has a good repeatability. ${ }^{\mathbf{8} 9}$ Synchronously, it is traditionally acknowledged that "Laba" garlic can be eaten when its colour turns green. The processing time plays an important role on the production of 
"Laba" garlic, but the most appropriate time to obtain "Laba" garlic has not been identified. Nevertheless, the traditional homemade processing of "Laba" garlic can remove the strong pungency of normal garlic, and characterize "Laba" garlic with an attractive green colour, faint spicy flavor, and sweet and sour taste. Innovative technology has been applied to the production of "Laba" garlic, which utilized dense phase carbon dioxide instead of vinegar soaking, the results of Dan et al. ${ }^{12}$ suggested that vinegar soaking was essential for obtaining "Laba" garlic with better flavor and taste. As far as we know, there are few reports on the composition of garlic soaked in vinegar, except Pure et al. ${ }^{16}$ who compared the constitutes of garlic fermented in red grape vinegar and Kombucha vinegar. Furthermore, soaking aged garlic in vinegar and making it into "Laba" garlic is one of the most effective means to reduce the intense pungent flavor of raw garlic, which also benefits for the market development and diversification of garlic products. Hence, it is still imperative to make fully understanding of chemical constituents of "Laba" garlic during traditional processing.

Recently, untargeted metabolomics approaches, which can detect the entire metabolites of samples, have been widely applied in different foods, such as doenjang, ${ }^{17}$ Cheonggukjang, ${ }^{18}$ and crab paste. ${ }^{19}$ In the meantime, these metabolomic analyses are powerful for comprehending metabolites associated with physiological and functional characteristics in foods during fermentation or processing.

Therefore, we investigated the whole chemical constituents influencing colour, flavor and physiological function of "Laba" garlic during traditional processing $(0,3,6,9,12,15,21,28$ and 42 days) in this study. GC-MS and HPLC-MS/MS were applied to discover broader classes of compounds, including volatiles, amino acids, organic acids, sugars and phenolic compounds. And correlations between metabolites and antioxidant activities of "Laba" garlic during traditional processing were also studied. This study can help us understand changes in the systemic compositions and physiological function of "Laba" garlic during its traditional processing. Furthermore, it can also provide new insight into the change of metabolic pathway in "Laba" garlic during traditional processing, and very helpful in its industrial production and process improvement.

\section{Material and methods}

\section{Chemicals agents and materials}

Chloroform $\left(\mathrm{CHCl}_{3}\right)$, $n$-hexane, methanol $(\mathrm{MeOH})$ and acetonitrile were supplied by Merck (Darmstadt, Germany). Normal alkanes standards (C7-C30), methoxyamine hydrochloride, $\mathrm{N}$ methyl- $N$-(trimethylsilyl) trifluoroacetamide (MSTFA), pyridine, formic acid, isoamyl alcohol, ribitol, gallic acid, potassium persulfate, hydrochloric acid $(\mathrm{HCl})$, methanoic acid, ferric chloride $\left(\mathrm{FeCl}_{3}\right)$, 1,1-diphenyl-2-picrylhydrazyl (DPPH), 2,2'azino-bis-3-ethylbenzothiazoline-cis-sulfonic acid (ABTS), 6hydroxy-2,5,7,8-tetramethylchroman-2-carboxylic acid (Trolox), 2,4,6-tripyridyl-s-triazine (TPTZ), Folin-Ciocalteu's phenol, acetic acid, and standards of phenolic compounds were obtained from Sigma-Aldrich (St. Louis, Missouri, USA). Solid phase microextraction (SPME) fiber coated with divinylbenzene/ carboxen/polydimethylsiloxane (DVB/CAR/PDMS, 50/30 $\mu \mathrm{m}$ ) was purchased from Supelco (Oakville, ON, Canada).

\section{Traditional processing of "Laba" garlic and sample preparation}

Garlic (Allium sativum L. 'Jinxiang') was harvested in June 2017 from Jinxiang county of China and collected directly from the producers, then stored the garlic at $4-8{ }^{\circ} \mathrm{C}$ for one month. Firstly, garlic bulbs with uniform size were chosen and then peeled, further washed three times with distilled water. Secondly, the drained garlic samples were packed into the jars and covered with vinegar $(\mathrm{pH}=3)$ (with acetic acid concentration of $36.89 \mathrm{mg} \mathrm{mL}^{-1}$-shaanxi Yuhong Food Group Co., Ltd, Hancheng, China) at room temperature. ${ }^{9}$ Then, the garlic samples were collected at 0 day prior to processing, and at 3, 6, $9,12,15,21,28$, and 42 days during traditional processing. Half of each garlic sample was immediately frozen in liquid $\mathrm{N}_{2}$ and dried using a freeze dryer to analyze primary metabolites, phenolic compounds and antioxidant activities. At the same time, the other half of the garlic sample was used for volatile analysis and colour measurement.

\section{GC-MS analysis of volatile metabolites}

Volatile metabolites in garlic were analyzed by the method described by Kimbaris et al. $^{20}$ and Priego-Capote et al. ${ }^{21}$ with slight modification. And the method can be described as follows: $2 \mathrm{~g}$ crushed sample was put into a screw-cap vial (20 $\mathrm{mL}$ ), then the vial was put on a CTC CombiPal autosampler (Zwingen, Switzerland) coupled with an Agilent 7890A gas chromatograph of Agilent Technologies (Wilmington, USA). A SPME fiber $(2 \mathrm{~cm})$ coated with a DVB/CAR/PDMS $(50 / 30 \mu \mathrm{m})$ layer was used because of its best response for the garlic volatiles. $^{22}$ The automated sample preparation processing was described as follows: the vial was kept at $40{ }^{\circ} \mathrm{C}$ for $10 \mathrm{~min}$, then the fiber was inserted into the vial headspace, following heating the vial at $40{ }^{\circ} \mathrm{C}$ for $40 \mathrm{~min}$ with continuous stirring. Finally, the volatile were desorbed in the GC inlet at $250{ }^{\circ} \mathrm{C}$ for $5 \mathrm{~min}$ (split ratio $5: 1$ ) and the fiber was reconditioned for $2 \mathrm{~min}$ at $270^{\circ} \mathrm{C}$. A HP-Innowax $(60 \mathrm{~m} \times 0.25 \mathrm{~mm} \times 0.25 \mu \mathrm{m}$, Agilent Technologies, USA) column was used. The oven temperature program was $40{ }^{\circ} \mathrm{C}$ for $3 \mathrm{~min}, 5{ }^{\circ} \mathrm{C}$ per minute to $240{ }^{\circ} \mathrm{C}$, and kept $240{ }^{\circ} \mathrm{C}$ for $5 \mathrm{~min}$, a post run at $300{ }^{\circ} \mathrm{C}$ for $2 \mathrm{~min}$. Helium was used as carrier gas at a constant flow rate of $1.0 \mathrm{~mL} \mathrm{~min}^{-1}$. The temperatures of MS quadrupole and transfer line of MSD detector (5975C inert XL MSD with Triple-Axis Detector, Agilent) were operated at $150{ }^{\circ} \mathrm{C}$. Electron ionization (EI) was used at $70 \mathrm{eV}$, MS ion source was $230^{\circ} \mathrm{C}$ and the scanning mass range was $40-500 \mathrm{~m} / \mathrm{z}$. For volatile analysis, six biological replications were required with a randomized injection order.

\section{GC-MS analysis of primary metabolites}

Primary metabolite analysis was carried out using the method of Lisec et $a .^{23}$ Each dried sample (100 mg) was extracted using $60 \%$ aqueous methanol $\left(1400 \mu \mathrm{L}\right.$, pre-cooled at $\left.-20{ }^{\circ} \mathrm{C}\right)$ with ribitol $\left(60 \mu \mathrm{L}, 0.2 \mathrm{mg} \mathrm{mL}^{-1}\right.$ stock in distilled $\left.\mathrm{H}_{2} \mathrm{O}\right)$ as internal standard. The sample was homogenized under an ultrasonic 
treatment process at $30 \mathrm{~Hz}$ for $10 \mathrm{~min}$ using a sonicator (Fisher Scientific Inc., Pittsburgh, PA). Then, the sample was shaken at $70{ }^{\circ} \mathrm{C}$ for $10 \mathrm{~min}$ in a thermomixer $(950 \mathrm{rpm})$. After centrifugation (11000 g, $4{ }^{\circ} \mathrm{C}, 15 \mathrm{~min}$ ) (small bench top centrifuge, Sigma 1-14, Germany), the supernatant was transferred to a new glass vial with addition of chloroform $(750 \mu \mathrm{L}$, pre-cooled at -20 $\left.{ }^{\circ} \mathrm{C}\right)$ and distilled $\mathrm{H}_{2} \mathrm{O}\left(1500 \mu \mathrm{L}, 4{ }^{\circ} \mathrm{C}\right)$, and then vortexed for $10 \mathrm{~s}$. After centrifuging the sample again, the supernatant was filtered through a $0.2 \mu \mathrm{m}$ polytetrafluoroethylene (PTFE) filter, and dried using a speed vacuum concentrator (Eppendorf AG, Hamburg, Germany) without heating. The oximation was carried out by adding $40 \mu \mathrm{L}$ of methoxyamine- $\mathrm{HCl}\left(20 \mathrm{mg} \mathrm{mL} \mathrm{m}^{-1}\right.$ in pyridine) to dried extracts at $37^{\circ} \mathrm{C}$ for $90 \mathrm{~min}$. Then, $70 \mu \mathrm{L}$ of MSTFA was added to the sample at $37^{\circ} \mathrm{C}$ for $30 \mathrm{~min}$. Primary metabolite measurement was accomplished by another GC system (Agilent7890A, Palo Alto, USA) equipped with an autosampler (Agilent 7693A), and connected with a MSD detector (5975C inert XL MSD with Triple-Axis Detector, Agilent). The final sample $(1 \mu \mathrm{L})$ was injected into the GC injection port (split ratio of $10: 1$ ) immediately (the front inlet temperatures was $250{ }^{\circ} \mathrm{C}$ ). Metabolites separation was performed on a DB-5MS capillary column $(30 \mathrm{~m} \times 0.25 \mathrm{~mm} \times 0.25$, Agilent Technologies, USA), with helium gas at a constant flow rate of 1.5 $\mathrm{mL} \min ^{-1}$. The oven temperature program was $70{ }^{\circ} \mathrm{C}$ for $2 \mathrm{~min}$, $10{ }^{\circ} \mathrm{C}$ per minute to $300^{\circ} \mathrm{C}$, and kept $300{ }^{\circ} \mathrm{C}$ for $6 \mathrm{~min}$, a post run at $310{ }^{\circ} \mathrm{C}$ for $4 \mathrm{~min}$. The temperatures of $\mathrm{MS}$ quadrupole and transfer line were operated at $150{ }^{\circ} \mathrm{C}$. Electron ionization (EI) was used at $70 \mathrm{eV}$, MS ion source was $230{ }^{\circ} \mathrm{C}$ and the scanning mass range was $40-600 \mathrm{~m} / \mathrm{z}$. For primary metabolite analysis, six biological replications were performed with a randomized injection order.

\section{HPLC-MS/MS analysis of phenolic compounds}

Each dried sample (100 mg) was extracted using $1500 \mu \mathrm{L}$ of $60 \%$ aqueous methanol. The sample was homogenized and sonicated at $30 \mathrm{~Hz}$ for 10 min using a sonicator (Fisher Scientific Inc., Pittsburgh, PA). After centrifugation (11000 g, $4{ }^{\circ} \mathrm{C}, 15 \mathrm{~min}$ ) (small bench top centrifuge, Sigma 1-14, Germany), the supernatant was filtered through a $0.2 \mu \mathrm{m}$ PTFE filter. Analysis was performed using a HPLC (Symbiosis Pharma, Spark, Plainsboro, NJ, USA) with an MS/MS (API 6500QTRAP; Applied Biosystems/MDS Sciex), and a ZORBAX SB-C18 column $(150 \mathrm{~mm}$ $\times 4.6 \mathrm{~mm}$ i.d., $5 \mu \mathrm{m}$ particle size, Agilent, Santa Clara, USA). The mobile phases consisted of $0.1 \% \mathrm{v} / \mathrm{v}$ formic acid in water (solvent A) and acetonitrile (solvent $\mathrm{B}$ ). The injection volume was $10 \mu \mathrm{L}$ and the flow rate was $0.8 \mathrm{~mL} \min ^{-1}$. The gradient elution was as follows: $10 \% \mathrm{~B}(0-2 \mathrm{~min}), 10-60 \% \mathrm{~B}(2-7 \mathrm{~min})$, 60-80\% B (7-15 min), 80-10\% B (15-16 min), and 10\% B (16-17 $\min )$. The ion spray voltage was $4500 \mathrm{~V}$ in negative ionization mode. Nitrogen was used as nebulizer gas and curtain gas, which were set at 55 and 40 psi, respectively. Auxiliary heating gas was set at 55 psi $550{ }^{\circ} \mathrm{C}$. Multiple reactions monitoring (MRM) mode was used for acquiring data, and detailed information of the 15 phenolic compounds was shown in Table S3. $\dagger$ For phenolic compounds metabolites analysis, six biological replications were executed with a randomized injection order.

\section{Evaluation of antioxidant capacity}

\section{DPPH radical scavenging assay}

The extraction method was according to the method of HPLCMS/MS analysis. DPPH assay was executed on a 96-well microplate and based on a literature method. ${ }^{24} 20 \mu \mathrm{L}$ aliquot of sample extract and $180 \mu \mathrm{L}$ of DPPH in methanol solution (200 $\mu \mathrm{M})$ were mixed in a 96-well microplate. The mixture was incubated at $37{ }^{\circ} \mathrm{C}$ for $30 \mathrm{~min}$, and a spectrophotometer (Multiskan GO, Thermo Scientific, USA) was utilizing to measure the absorbance at $515 \mathrm{~nm}$. The results were calculated using a standard curve (0.05-0.8 $\mathrm{mM}$ Trolox) and presented as mmol equivalent Trolox/mg dry weight.

\section{ABTS radical scavenging assay}

ABTS radical scavenging assay was performed using the modified procedures of Re et al. ${ }^{25}$ A $7 \mathrm{mM}$ ABTS solution was prepared by reacting $0.0768 \mathrm{~g}$ ABTS salt with $0.0132 \mathrm{~g}$ potassium persulfate in $20 \mathrm{~mL}$ deionized water, then placed the mixture in dark for $16 \mathrm{~h}$. A certain amount of deionized water was added to the $7 \mathrm{mM}$ ABTS solution to obtain $0.7 \pm 0.02$ absorbance of working solution at $734 \mathrm{~nm} .180 \mu \mathrm{L}$ of ABTS working solution and $20 \mu \mathrm{L}$ aliquot of sample extract were reacted in a 96-well microplate at room temperature for $6 \mathrm{~min}$, following recording the absorbance at $734 \mathrm{~nm}$ by a spectrophotometer. The estimation of ABTS value was using a Trolox standard (0.01 to 0.1 $\mathrm{mM})$.

\section{Ferric reducing power (FRAP) assay}

The FRAP stock solutions was prepared by mixing $10 \mathrm{mM}$ TPTZ in $40 \mathrm{mM} \mathrm{HCl}, 20 \mathrm{mM} \mathrm{FeCl}{ }_{3} \cdot 6 \mathrm{H}_{2} \mathrm{O}$, and $300 \mathrm{mM}$ acetate buffer (pH 3.6). $175 \mu \mathrm{L}$ of FRAP reagent and $25 \mu \mathrm{L}$ of sample extract in a 96-well microplate reacted at room temperature for $10 \mathrm{~min}$, following measuring its absorbance at $593 \mathrm{~nm}$. The FRAP value of the sample solution was calculated by a Trolox standard $(0.01$ to $0.4 \mathrm{mM}$ ).

\section{Measurement of garlic discolouration}

The garlic discolouration was measured according to the method of Dan et $a .^{12} 50 \mathrm{~g}$ garlic samples were homogenized with $50 \mathrm{~mL}$ anhydrous methanol by a beater (JYDZ-31B; Jiuyang Co. Ltd., Shandong, China), then centrifuging (10000 g) at $4{ }^{\circ} \mathrm{C}$ for $10 \mathrm{~min}$. Absorbance at $590 \mathrm{~nm}$ and $440 \mathrm{~nm}$ of the supernatant was measured using a spectrophotometer (Multiskan GO, Thermo Scientific, USA).

\section{Data processing and statistical analysis}

For the data of GC-MS, the raw data were imported to MetAlign software (http://www.metalign.nl) and transformed to NetCDF format (*.cdf) files, following the baseline correction, peak detection, and alignment. The volatile metabolites were identified by matching the mass spectra to the NIST mass spectral library (http://www.nist.gov), while the primary metabolites as TMS derivatives were identified with the NIST mass spectral library (http://www.nist.gov) and the Golm Metabolome 
Database (http://www.csbdb.mpimp-Golm.mpg.de/csbdb/gmd/ home/gmd_sm. html). According to the method of Kovats, ${ }^{26}$ tentative identification of metabolites was performed by using C7-C30 alkanes to calculate the retention indices. Some important metabolites were confirmed using authentic standards, including diallyl sulfide, diallyl disulfide, diallyl trisulfide, L-alanine, s-leucine, DL-malic acid, s-proline, L-tyrosine, Dfructose and D-glucose. A single ion fragment of each compound was selected for the quantification. Relative intensity of volatile metabolite was obtained based on the ratios between the peak area of a particular component in the sample and the total peak area of all components in the sample. While relative concentration of the primary metabolite was calculated using ribitol as internal standard. The normalized data was used for partial least-squares discriminant analysis (PLS-DA) with the software SIMCA- $\mathrm{P}^{+}$(version 13.0, Umerics, Umea, Sweden). For the phenolic compounds obtained by HPLC-MS/MS, Analyst software (version 1.6.2) was used to quantify the target compounds based on the external standard curve methods. In addition, Duncan's multiple-range test $(p<0.05)$ was carried out using SPSS software (version 20, IBM Inc. USA), to determine the significance of differences among the metabolites and antioxidant activities in garlic samples, according to different traditional processing times.

\section{Result and discussion}

\section{Colour analysis of "Laba" garlic during processing}

Previous reports suggested that the green colour of "Laba" garlic is generated by the combination of the blue and yellow pigments. ${ }^{8}$ The two pigments have similar structures and were generated through parallel processes. ${ }^{27}$ And concentrations of these two pigments correspond to the absorbance at $590 \mathrm{~nm}$ and $440 \mathrm{~nm}$, respectively. ${ }^{9-11}$ Therefore, absorbance values at $590 \mathrm{~nm}$ (blue pigments) and $440 \mathrm{~nm}$ (yellow pigments) of the garlic extract were measured to monitor the colour changes of "Laba" garlic during processing. In this study, the colour change of "Laba" garlic during processing is consistent with the result obtained from Bing et al. ${ }^{8}$ As shown in Fig. 1, the colour of garlic samples during traditional processing changed from white to green and then to yellow. And the strongest green
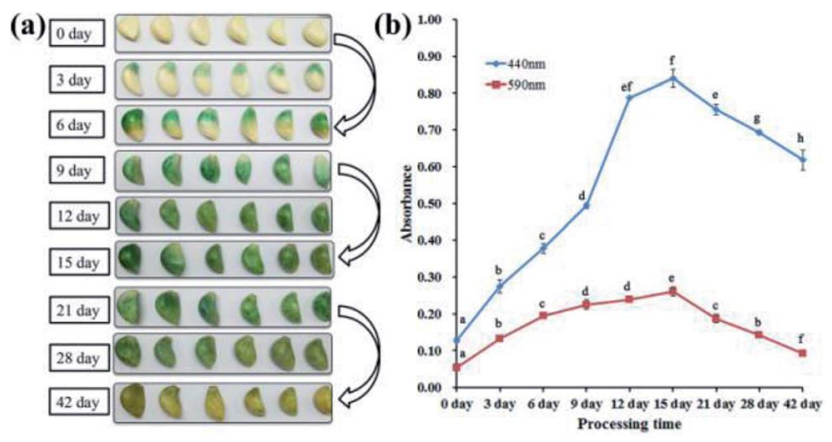

Fig. 1 Colour of "Laba" garlic (a) and absorbance of garlic extract (b) according to different processing times $(n=3)$. Different letters indicate significant differences between samples (Duncan test $p<0.05$ ). colour of garlic sample was observed at day 15 , and then began to fade (Fig. 1a). In response to this, the absorbance of blue and yellow pigments performed the similar change pattern, which increased gradually from day 0 to day 15 and reached the maximum at day 15 , then suffered a decrease until day 42 (Fig. 1b). Moreover, it is worth noting that the absorbance of blue pigments at $590 \mathrm{~nm}$ decreased $64.8 \%$ from day 15 to day 42 , while the absorbance of yellow pigments at $440 \mathrm{~nm}$ decreased $26.4 \%$. And this result can explain the change of green colour to yellow in "Laba" garlic from day 15 to day 42 .

\section{Volatile and primary metabolite analysis of "Laba" garlic during processing}

Volatile and primary metabolites of "Laba" garlic during traditional processing $(0,3,6,9,12,15,21,28$ and 42 days) were analyzed by GC-MS. A total of 44 volatile metabolites and 36 primary metabolites were recognized after data preprocessing and compound identification (Tables S1 and S2 $\dagger$ ). The volatile metabolites contained 26 organosulfur compounds, which were mainly derived from $S$-alk(en)yl-L-cysteine, while the 18 nonorganosulfur compounds were largely organic acids and esters. Moreover, primary metabolites consisted of 19 amino acids, 6 organic acids, 11 sugars and sugar derivatives.

In order to discriminate the samples at different processing times and explore variation of metabolites, PLS-DA models were established based on volatile and primary metabolites, respectively. And the obtained results were shown in Fig. 2. The model quality based on volatile metabolites was evaluated with $R^{2} X$ $($ cum $)=0.996, R^{2} Y$ (cum) $=0.843$, and $Q^{2}$ (cum) $=0.815$, while the model based on primary metabolites was assessed with $R^{2} X$ (cum) $=0.987, R^{2} Y(\mathrm{cum})=0.844$, and $Q^{2}$ (cum) $=0.823$. The internal validation of the PLS-DA model was accomplished by a permutation test $(n=200$, intercepts for volatile metabolites:
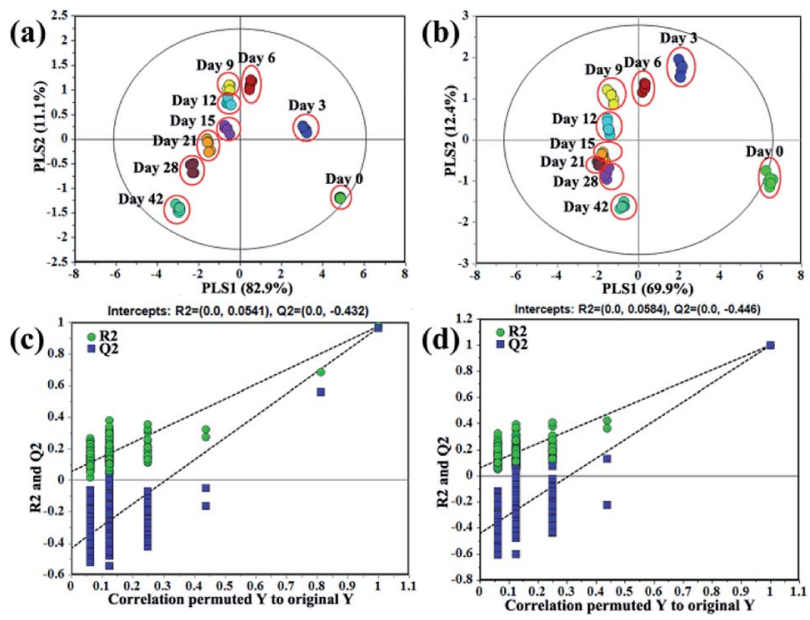

Fig. 2 Partial least-squares discriminant analysis (PLS-DA) scores and their permutation test plots (200 permutation tests) for garlic samples according to different processing times. (a) Score plot based on volatile metabolites, (b) score plot based on primary metabolites, (c) permutation test plots of PLS-DA model based on volatile metabolites, (d) permutation test plots of PLS-DA model based on primary metabolites. 
$R 2=0.0541, Q 2=-0.0432$; intercepts for primary metabolites: $R 2=0.0584, Q 2=-0.0446$ ), which indicated that the PLS-DA models were all statistically acceptable. Moreover, it can be obviously seen that the score plot of volatile metabolites presented a similar discrimination tendency with that of primary metabolites (Fig. 2a and b). The samples at the initial and later processing times ( 0,3 and 42 days) were more clearly distinguished from the other processing times $(6,9,12,15,21$ and 28 days).

In the volatile profile of garlic samples, 20 volatile metabolites were selected as notable changed metabolites during different processing times according to variable importance in the projection (VIP) $>0.7$ and the statistical significance $(p<0.05)$ (Table 1). The change behaviors of these metabolites during processing times were clearly presented in the heat map (Fig. 3a), in which values represent fold changes normalized by an average of all values according to different processing times. As compared to the unprocessed garlic ( 0 day), most organosulfur compounds showed a significant change from day 3 . This change could be explained with the damage of garlic cell membrane by vinegar. According to the report of Bing et al., ${ }^{8,9}$ the permeability of both plasma membrane and intracellular membrane of garlic are improved after the garlic being soaked in vinegar. Thus, alliin and alliinase in garlic react rapidly to form allicin. However, allicin is unstable and highly reactive, which can be easily transformed into a series of organosulfur compounds. It is worth noting these organosulfur compounds are thought to be the main sources of the biological properties and the particular pungent flavor of fresh garlic. ${ }^{28,29}$ Besides, the odor differences between different garlics or garlic products may be largely attributed to the type and contents of these organosulfur compounds. In this study, three dissimilar patterns of these organosulfur compounds during traditional processing were observed: high concentrations at the initial time ( 0 day), around early times ( 3 to 15 day), and the later times ( 21 to 42 day). The levels of 5 organosulfur compounds including diallyl tetrasulphide, 3-vinyl-1,2-dithiacyclohex-4-ene, 3,4-dimethylthiophene, sulfur dioxide, and trans-3,5-diethyl-1,2,4-trithiolane were high at the initial time ( 0 day), then decreased gradually until day 42 . Five organosulfur compounds: allyl methyl disulfide, diallyl disulfide, 1,3-dithiole-2-thione, propyl ethynyl sulfoxide, and methyl propenyl disulfide, were high around early times ( 3 to 15 day). At the later times ( 21 to 42 day), 4 organosulfur compounds: allyl methyl sulfide, allyl methyl trisulfide, diallyl sulfide, and diallyl trisulfide presented a gradual increase. The major organosulfur compounds including diallyl disulfide, diallyl tetrasulphide and 3-vinyl-1,2-dithiacyclohex-4-ene exhibited a gradually decreasing trend from day 3 to day 42 . As shown in Fig. $3 \mathrm{~b}$, the relative concentration of organosulfur compounds also progressively decreased from day 3 to day 42. Decreased content of these organosulfur compounds in the volatile metabolites may own to their diffusion from garlic cloves to vinegar along with the traditional processing, ${ }^{9}$ which is also the main reason for the formation of the faint spicy flavor of "Laba" garlic.

Regarding to the non-organosulfur compounds of "Laba" garlic, acetic acid, butanoic acid, and benzoic acid, ethyl ester

Table 1 Notable changed volatile metabolites identified in garlic

\begin{tabular}{|c|c|c|c|c|c|c|c|c|}
\hline No. & $\mathrm{RT}^{a}(\min )$ & Compound name ${ }^{b}$ & $\begin{array}{l}\text { Targetted ion } \\
(\mathrm{m} / \mathrm{z})\end{array}$ & MS fragment ion $(\mathrm{m} / \mathrm{z})$ & $\mathrm{ID}^{c}$ & $\mathrm{RI}^{d}$ & $\mathrm{VIP}^{e}$ & $p$-Value ${ }^{f}$ \\
\hline \multicolumn{9}{|c|}{ Organosulfur compounds } \\
\hline 1 & 6.26 & Sulfur dioxide & 64 & $16,32,48,64$ & MS/NIST & 870.25 & 1.743 & $8.626 \times 10^{-9}$ \\
\hline 2 & 6.71 & Propyl ethynyl sulfoxide & 43 & $27,39,43,74$ & MS/NIST & 895.36 & 1.480 & $3.080 \times 10^{-11}$ \\
\hline 3 & 6.73 & Trans-3,5-Diethyl-1,2,4-trithiolane & 74 & $39,41,45,59,74,180$ & MS/NIST & 896.20 & 1.141 & $7.111 \times 10^{-11}$ \\
\hline 4 & 8.19 & Allyl methyl sulfide & 88 & $39,41,45,61,73,88$ & STD & 966.23 & 0.899 & $2.683 \times 10^{-6}$ \\
\hline 5 & 13.51 & Diallyl sulfide & 45 & $39,41,45,73,99,114$ & STD & 1157.47 & 1.340 & $1.307 \times 10^{-8}$ \\
\hline 6 & 16.73 & 3,4-Dimethylthiophene & 111 & $39,45,97,111$ & MS/NIST & 1265.61 & 0.722 & $3.323 \times 10^{-5}$ \\
\hline 7 & 17.55 & Allyl methyl disulfide & 120 & $39,41,45,73,79,88,120$ & STD & 1291.67 & 1.426 & $2.750 \times 10^{-8}$ \\
\hline 8 & 17.77 & Methyl propenyl disulfide & 73 & $41,45,72,73,87,120$ & MS/NIST & 1298.29 & 0.866 & $9.379 \times 10^{-13}$ \\
\hline 9 & 23.25 & Diallyl disulfide & 41 & $39,41,73,81,113,146$ & STD & 1499.91 & 2.296 & $7.191 \times 10^{-8}$ \\
\hline 10 & 23.35 & Diallyl tetrasulphide & 73 & $39,41,64,73,105,146$ & MS/NIST & 1504.51 & 1.499 & $4.115 \times 10^{-14}$ \\
\hline 11 & 26.01 & Allyl methyl trisulfide & 73 & $39,41,73,79,87,105,114,152$ & STD & 1610.54 & 1.199 & $1.576 \times 10^{-25}$ \\
\hline 12 & 29.56 & 3-Vinyl-1,2-dithiacyclohex-4-ene & 111 & $45,71,77,97,103,111,144$ & MS/NIST & 1764.77 & 1.927 & $4.445 \times 10^{-4}$ \\
\hline 13 & 30.65 & Diallyl trisulfide & 113 & $39,41,45,73,113,178$ & STD & 1813.52 & 1.728 & $8.430 \times 10^{-12}$ \\
\hline 14 & 44.60 & 1,3-Dithiole-2-thione & 58 & $32,44,58,76,90,134,136$ & MS/NIST & 2550.76 & 0.712 & $1.193 \times 10^{-11}$ \\
\hline \multicolumn{9}{|c|}{ Non-organosulfur compounds } \\
\hline 15 & 3.92 & Propene & 41 & $41,42,40,39,27,15$ & MS/NIST & $\sim$ & 1.531 & $1.823 \times 10^{-4}$ \\
\hline 16 & 4.61 & Acetaldehyde & 29 & $15,29,43,44$ & MS/NIST & 719.62 & 0.919 & $4.204 \times 10^{-12}$ \\
\hline 17 & 21.98 & Acetic acid & 43 & $15,43,45,60$ & STD & 1461.68 & 2.268 & $3.438 \times 10^{-12}$ \\
\hline 18 & 24.47 & $(Z)$-Benzaldoxime & 103 & $39,51,76,103,121$ & MS/NIST & 1550.37 & 1.414 & $9.463 \times 10^{-17}$ \\
\hline 19 & 26.45 & Butanoic acid & 60 & $27,41,43,60,73,88$ & STD & 1630.30 & 0.812 & $6.644 \times 10^{-17}$ \\
\hline 20 & 27.71 & Benzoic acid, ethyl ester & 105 & $51,77,105,122,150$ & MS/NIST & 1682.85 & 0.791 & $1.755 \times 10^{-3}$ \\
\hline
\end{tabular}

${ }^{a}$ RT is retention time. ${ }^{b}$ Compounds were selected by VIP $>0.7$ and $p$-value $<0.05{ }^{c}$ Identification: STD, standard compound; MS/NIST, comparing mass fragment pattern within house library and NIST. ${ }^{d}$ RI is retention indices calculated with $n$-alkanes. ${ }^{e}$ VIP (variable importance in the projection) values were determined by PLS-DA. ${ }^{f} p$-Values were analyzed by ANOVA with Duncan's test. 


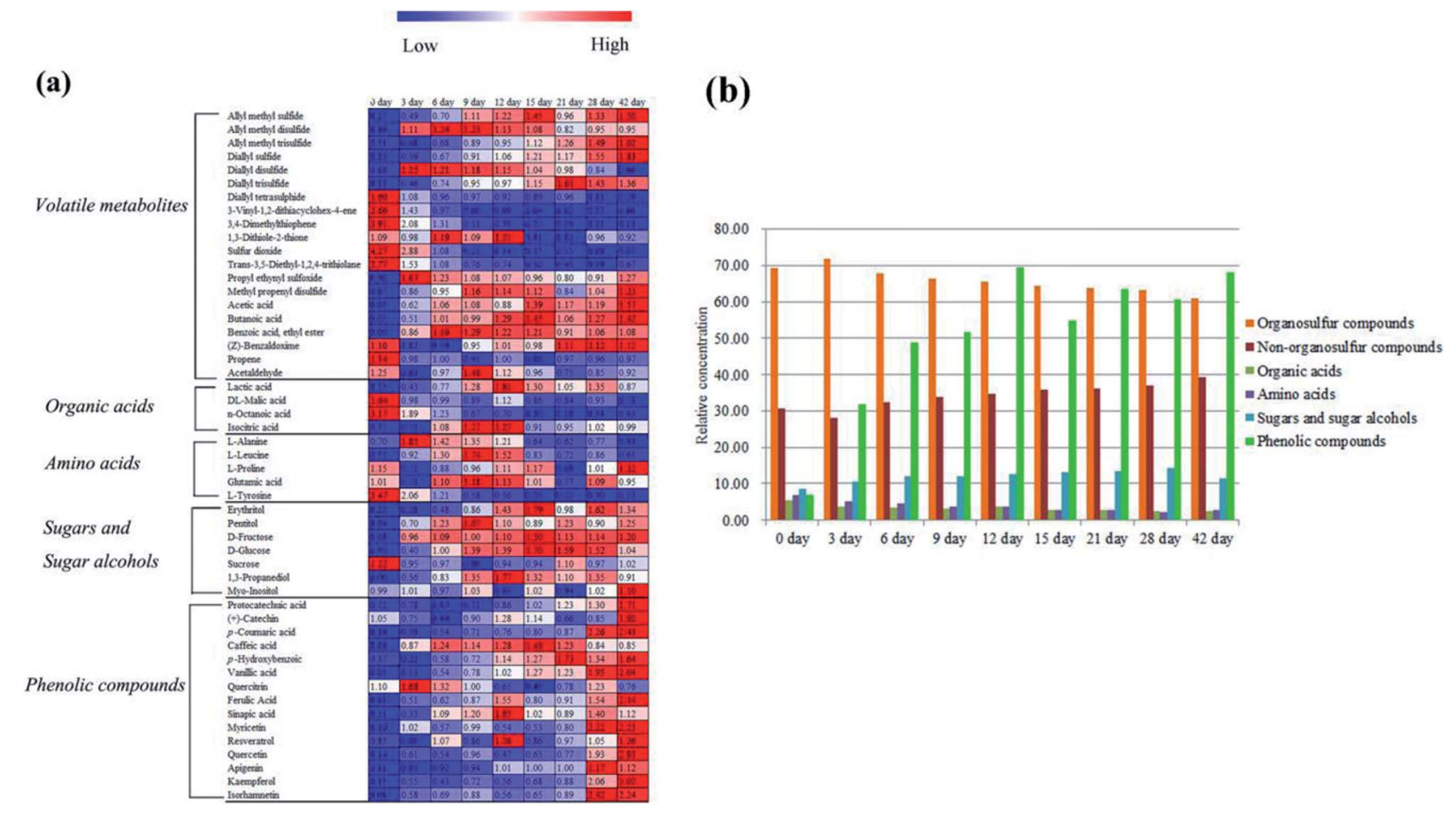

Fig. 3 (a) Heat-map of the contents of metabolites in garlic samples according to different processing times (values are fold changes normalized by an average of all values according to different processing times, colours represent relative intensities for each compound from dark green of low intensity to dark red of high intensity), (b) contents of metabolites (organosulfur compounds, non-organosulfur compounds, organic acids, amino acids, sugars and sugar alcohols, and phenolic compounds) in garlic samples according to different processing times.

were negligible at the initial time ( 0 day), then significantly increased at day 3 and presented higher concentrations during day 3 to day 42 . The relative content of non-organosulfur compounds in "Laba" garlic also presented an increasing trend during the whole processing (Fig. 3b), which may contribute to the flavor and taste of "Laba" garlic, and improve its palatability and aroma.

The sweet and sour taste "Laba" garlic mainly origins from its primary metabolites including organic acids, amino acids, sugars and sugar alcohols. Table 2 lists lactic acid, DL-malic acid, $n$-octanoic acid, isocitric acid, L-alanine, L-leucine, $\mathrm{L}^{-}$ proline, glutamic acid, L-tyrosine, erythritol, pentitol, D-fructose, D-glucose, sucrose, 1,3-propanediol and myo-inositol, which were referred as the significantly varied primary metabolites in garlic samples during different processing times (VIP $>0.7$ and $p<0.05)$. As shown in Fig. 3a, lactic acid and isocitric acid performed uniform change tendency, the levels of which were progressively increased from day 3 to day 12 and then decreased from day 15 to day 42 . Moreover, lactic acid, isocitric acid and DL-malic acid reached its maximum level at day 12 , and the changes in these organic acids may indicate that day 12 is a vital process time of "Laba" garlic. Acetic acid was the most abundant organic acid in vinegar and plays an important role during the traditional processing of "Laba" garlic. Increased content of acetic acid in the "Laba" garlic would reduce its intracellular $\mathrm{pH}$, and further influence several intracellular processes, such as nutrient transportation, synthesis of some compositions and change of cell structure. ${ }^{8}$ According to the proposed pathway
(Fig. 4) of primary metabolites which was modified from the KEGG database (http://www.genome.jp/kegg/), the increasing contents of lactic acid and isocitric acid before day 12 possibly resulted from the conversion of other intermediates during the glycolysis and TCA cycles. In addition, these organic acids mainly improve the acidity of "Laba" garlic as a flavoring agent. Along with the time of vinegar soaking, the permeability of garlic membrane can be increased, which suggests that more organic acids in their undissociated forms inside garlic cells would transfer into the vinegar, including lactic acid and isocitric acid. This may explain the content decrease of lactic acid and isocitric acid from day 15 to day 42 .

Furthermore, pervious investigations indicate that garlic is a good source of essential amino acids. ${ }^{30}$ As can be seen in Fig. 3a, L-alanine and L-leucine performed opposite trends from day 3 to day 12 , and both of them have been observed a pronounced reduction about $50 \%$ from day 12 to day 15 . A previous study found that L-alanine and L-leucine could react with di(1-propenyl) thiosulfinate to form pigments, and $\mathrm{L}^{-}$ alanine contributed more to the garlic greening. ${ }^{11}$ Thus, the notable reduction of $\mathrm{L}$-alanine and $\mathrm{L}$-leucine from day 12 to day 15 could be attributed to the pigments formation. L-proline increased from day 3 to day 15 and L-tyrosine appeared a decrease from day 0 to day 42 . Moreover, since no coloured compounds were formed when proline was added into thiosulfinate, ${ }^{15}$ the increase of L-proline probably attributed to the degradation of proteins of garlic under acidic conditions or produced from a few intermediates. Moreover, these amino 
Table 2 Notable changed primary metabolites identified in garlic samples during processing

\begin{tabular}{|c|c|c|c|c|c|c|c|c|}
\hline No. & $\mathrm{RT}^{a}(\min )$ & Compound name ${ }^{b}$ & $\begin{array}{l}\text { Targetted ion } \\
(\mathrm{m} / \mathrm{z})\end{array}$ & MS fragment ion $(\mathrm{m} / \mathrm{z})$ & $\mathrm{ID}^{c}$ & $\mathrm{RI}^{d}$ & $\mathrm{VIP}^{e}$ & $p$-Value $f^{f}$ \\
\hline 1 & 5.717 & 1,3 Propanediol & 130 & $66,73,115,130,147$ & MS/NIST & $\sim$ & 1.81 & $1.731 \times 10^{-7}$ \\
\hline 3 & 6.445 & L-Alanine & 147 & $73,116,147,190$ & MS/NIST & $\sim$ & 1.17 & $2.731 \times 10^{-18}$ \\
\hline 4 & 7.272 & L-Leucine & 86 & $73,75,86,103,146,188$ & STD & 1156.05 & 0.99 & $3.721 \times 10^{-12}$ \\
\hline 5 & 11.841 & DL-Malic acid & 133 & $73,133,147,189,233,245,335$ & STD & 1479.82 & 1.22 & $1.645 \times 10^{-14}$ \\
\hline 8 & 12.437 & Pentitol & 117 & $73,103,117,147,217,307$ & MS/NIST & 1527.58 & 0.90 & $5.196 \times 10^{-9}$ \\
\hline 9 & 12.95 & $n$-Octanoic acid & 147 & $73,147,186$ & MS/NIST & 1570.05 & 0.83 & $6.870 \times 10^{-15}$ \\
\hline 10 & 13.446 & Glutamic acid & 128 & $73,128,147,246,348$ & MS/NIST & 1610.35 & 0.82 & $3.129 \times 10^{-6}$ \\
\hline 11 & 15.267 & L-Tyrosine & 218 & $73,100,147,218,406$ & MS/NIST & 1772.14 & 0.76 & $4.151 \times 10^{-11}$ \\
\hline 12 & 15.631 & Isocitric acid & 273 & $73,147,273,347,363,375,465$ & MS/NIST & 1804.08 & 1.39 & $2.258 \times 10^{-21}$ \\
\hline 13 & 16.161 & D-Fructose & 217 & $73,103,147,217,307$ & STD & 1856.49 & 1.91 & $8.379 \times 10^{-6}$ \\
\hline
\end{tabular}

${ }^{a}$ RT is retention time. ${ }^{b}$ Compounds were selected by VIP $>0.7$ and $p$-value $<0.05 .{ }^{c}$ Identification: STD, standard compound; MS/NIST, comparing mass fragment pattern within house library and NIST. ${ }^{d}$ RI is retention indices calculated with $n$-alkanes. ${ }^{e}$ VIP (variable importance in the projection) values were determined by PLS-DA. ${ }^{f} p$-Values were analyzed by ANOVA with Duncan's test.

acids perhaps offer different taste for "Laba" garlic during different processing times. L-Alanine, L-proline and L-tyrosine provide a sweet taste, while L-leucine offers a bitter taste, and glutamic acid relates to a savory taste. ${ }^{31}$

With regard to sugars, the level of sucrose slightly decreased during day 0 to day 9 as showed in Fig. 4 . While the content of D-fructose and D-glucose kept increasing before day 15 , which then decreased until day 42 . Both D-fructose and Dglucose showed obvious increase before day 15, probably resulting from the degradation of polysaccharides. According to the investigation of Chandrashekara et al., ${ }^{32}$ fructan is the main polysaccharide in garlic and fructans from aged garlic extract present obvious immunomodulatory activity. ${ }^{33}$ Meanwhile, previous studies found that fructose, glucose and sucrose can be obtained by the decomposition of fructan in garlic, especially under acidic condition. ${ }^{34}$ Similar change patterns were discovered with erythritol and pentitol, both of which increased from day 0 , then reached maximum at day 15

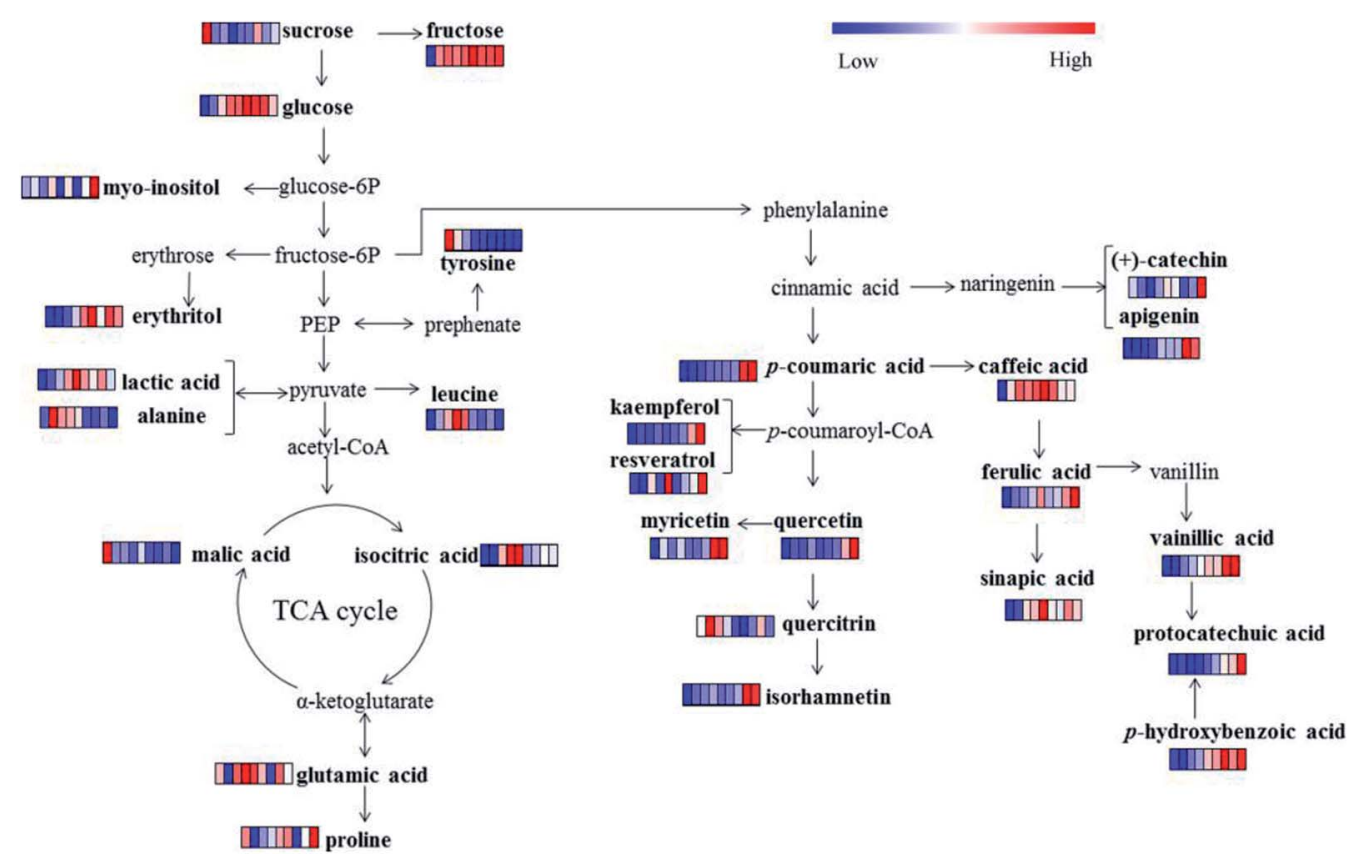

Fig. 4 The proposed metabolic pathway of primary metabolites and phenolic compounds in garlic samples during processing $(0,3,6,9,12,15$, 21, 28 and 42 days). Values are fold changes normalized by an average of all values according to different processing times. Colours represent relative intensities for each compound from dark green (low intensity) to dark red (high intensity). 
and day 9, respectively. We also discovered a decreasing trend among D-glucose and erythritol from day 28 to 42 , which suggested that the sweet taste of garlic may be reduced when the green colour transferred to yellow colour.

As seen in Fig. 3b, relative concentrations of detected primary metabolites (organic acids, amino acids, sugars and sugar alcohols) showed different changes during traditional processing of "Laba" garlic. In general, the concentration of amino acids decreased gradually from day 0 to day 28, whereas the levels of sugars and sugar alcohols increased during this time. Organic acids showed a decrease trend from day 0 to day 9 and from day 12 to day 42, respectively, and the maximum concentration was at day 12 . The content change of organic acids suggests that day 12 may be an important processing time of "Laba" garlic.

\section{Phenolic compounds analysis of "Laba" garlic during processing}

It has been well known that garlic is abundant of phenolic compounds, most of which exhibit a variety of biochemical properties, such as antioxidant activity, antifungal activity and inhibition of ACE activity. ${ }^{35,36}$ In this study, the screening of phenolic compounds in "Laba" garlic was based on a number of investigations about the major phenolic compounds in garlic, ${ }^{37-39}$ and then the possible phenolic compounds were identified with authentic standards. Thus, 7 phenolic acids and 8 flavonoids were detected and identified as remarkable phenolic compounds in garlic samples $(p<0.05$, Table 3$)$. According to Fig. $3 \mathrm{a}$ and $\mathrm{b}$, most phenolic compounds presented an increase trend during processing time, and the concentration of the 15 phenolic compounds from day 3 to day 42 was notably higher than the raw garlic ( 0 day). These results probably owed to the bioconversion of the conjugated forms of phenolic compounds into their free forms by acetic acid. ${ }^{40}$ Bound forms of phenolic compounds can be translated into free forms in many food processes, such as fermentation, malting, extrusion cooking, alkaline hydrolysis and acid hydrolyses. ${ }^{\mathbf{4 1 4 2}}$ In this research, vinegar provides a low $\mathrm{pH}$ that may break glycosidic bonds of phenolic compounds and sugars, and enhance the liberating of phenolic compounds, further leading to the increase in the content of phenolic compounds. ${ }^{43}$ Compared with flavonoids, phenolic acids have much higher concentrations such as ferulic acid, caffeic acid and sinapic acid (Table S4†). While relative higher levels of these three phenolic compounds were observed at day 12 or day 15. In Fig. 3b, the level of the 15 phenolic compounds obviously increased from day 0 and reached its maximum value at day 12. Therefore, the change behaviors of phenolic compounds indicate that day 12 may be the key processing time of "Laba" garlic. On the other hand, dietary intake of flavonoids in flavonoid-rich foods has presented benefits to human health, ${ }^{\mathbf{4 4}}$ which implies the potential nutritional value of "Laba" garlic with higher content of phenolic compounds at day 12.

Fig. 4 illustrated the metabolic pathways of the 15 phenolic compounds in "Laba" garlic according to the phenylpropanoid biosynthesis pathway, ${ }^{45}$ which provides a detailed transformation relationship between these phenolic compounds. For example, one part of cinnamic acid can be transformed into (+)-catechin and apigenin, another part of cinnamic acid is hydroxylated into $p$-coumaric acid and further converted into a number of phenolic compounds, such as caffeic acid, ferulic acid and quercitrin. Among the 15 phenolic compounds, caffeic acid and ferulic acid were the major phenolic acids in "Laba" garlic, which are known for their anti-oxidation characteristics and have shown to inhibit human low-density lipoprotein oxidation. ${ }^{\mathbf{4 6 , 4 7}}$ Besides exhibiting biological activities, the report of Zhou et al. ${ }^{48}$ also suggested that phenolic compounds isolated from garlic could affect the generation of sulfurcontaining compounds during garlic processing, which further influence the flavor and physiological functions of garlic. However, the relationship between phenolic compounds and characteristics of "Laba" garlic needs further study.

In addition, we conclude that there are at least three reasons for the change of metabolites present in the "Laba" garlic during processing. Firstly, the increased permeability of

Table 3 Notable changed phenolic compounds identified in garlic samples during processing

\begin{tabular}{|c|c|c|c|c|c|c|c|}
\hline No. & Name & $\mathrm{RT}^{a}(\min )$ & Molecular formula & Actual mass & Parent ion $(m / z)$ & MS fragments $(\mathrm{m} / \mathrm{z})$ & $p$-Value ${ }^{b}$ \\
\hline 1 & Protocatechuic acid & 5.33 & $\mathrm{C}_{7} \mathrm{H}_{6} \mathrm{O}_{4}$ & 154.12 & 153.00 & $109.1,90.9$ & $3.88 \times 10^{-7}$ \\
\hline 2 & $(+)$-Catechin & 5.77 & $\mathrm{C}_{15} \mathrm{H}_{14} \mathrm{O}_{6}$ & 290.27 & 289.00 & $122.9,245$ & $8.83 \times 10^{-5}$ \\
\hline 3 & $p$-Coumaric acid & 6.21 & $\mathrm{C}_{9} \mathrm{H}_{8} \mathrm{O}_{3}$ & 164.16 & 163.00 & $64.8,118.9$ & $5.96 \times 10^{-14}$ \\
\hline 4 & Caffeic acid & 6.22 & $\mathrm{C}_{9} \mathrm{H}_{8} \mathrm{O}_{4}$ & 180.16 & 179.00 & $134.9,117$ & $1.49 \times 10^{-13}$ \\
\hline 5 & p-Hydroxybenzoic & 6.22 & $\mathrm{C}_{7} \mathrm{H}_{6} \mathrm{O}_{3}$ & 138.12 & 137.00 & $92.9,65$ & $6.93 \times 10^{-12}$ \\
\hline 6 & Vanillic acid & 6.22 & $\mathrm{C}_{8} \mathrm{H}_{8} \mathrm{O}_{4}$ & 168.15 & 167.10 & $107.9,123.1$ & $8.79 \times 10^{-13}$ \\
\hline 7 & Quercitrin & 6.66 & $\mathrm{C}_{21} \mathrm{H}_{20} \mathrm{O}_{11}$ & 448.38 & 447.10 & $254.8,300.6$ & $2.20 \times 10^{-11}$ \\
\hline 8 & Ferulic acid & 7.10 & $\mathrm{C}_{10} \mathrm{H}_{10} \mathrm{O}_{4}$ & 194.18 & 193.10 & 134,149 & $3.51 \times 10^{-14}$ \\
\hline 9 & Sinapic acid & 7.11 & $\mathrm{C}_{11} \mathrm{H}_{12} \mathrm{O}_{5}$ & 224.21 & 222.90 & $163.9,148.9$ & $5.87 \times 10^{-6}$ \\
\hline 10 & Myricetin & 7.33 & $\mathrm{C}_{15} \mathrm{H}_{10} \mathrm{O}_{8}$ & 318.24 & 317.10 & $178.9,150.9$ & $3.99 \times 10^{-15}$ \\
\hline 11 & Resveratrol & 7.99 & $\mathrm{C}_{14} \mathrm{H}_{12} \mathrm{O}_{3}$ & 228.24 & 227.00 & $143,184.9$ & $5.84 \times 10^{-4}$ \\
\hline 12 & Quercetin & 8.00 & $\mathrm{C}_{15} \mathrm{H}_{10} \mathrm{O}_{7}$ & 302.24 & 301.20 & $150.9,178.8$ & $7.93 \times 10^{-14}$ \\
\hline 13 & Apigenin & 8.44 & $\mathrm{C}_{15} \mathrm{H}_{10} \mathrm{O}_{5}$ & 270.24 & 269.00 & 117,151 & $8.44 \times 10^{-16}$ \\
\hline 14 & Kaempferol & 8.44 & $\mathrm{C}_{15} \mathrm{H}_{10} \mathrm{O}_{6}$ & 286.24 & 285.20 & $187,158.5$ & $4.14 \times 10^{-8}$ \\
\hline 15 & Isorhamnetin & 8.65 & $\mathrm{C}_{16} \mathrm{H}_{12} \mathrm{O} 7$ & 316.26 & 315.00 & $300,150.8$ & $1.69 \times 10^{-5}$ \\
\hline
\end{tabular}

${ }^{a} \mathrm{RT}$ is retention time. ${ }^{b} p$-Values were analyzed by ANOVA with Duncan's test. 
membrane structure of garlic cells at the early processing times can promote the entry of vinegar to the interior of the garlic, which would lead to the degradation of macromolecular and improve the content of small molecules, such as nonorganosulfur compounds, organic acids, amino acids, sugars and sugar alcohols, and phenolic compounds. Secondly, the structure breakdown of garlic cell membrane at the later processing times may result in the transfer of garlic compositions to vinegar. Thirdly, a number of compounds may be involved in the discolouration of "Laba" garlic during processing.

\section{Correlations between metabolites and antioxidant activities of "Laba" garlic during processing}

In this study, antioxidant activities of "Laba" garlic samples during processing were measured by using DPPH, ABTS and FRAP assay. As shown in Table 4, the data results from DPPH, FRAP and ABTS represented similar change tendency during the whole processing, which generally decreased from day 0 to day 28. Among all the tested samples, the highest antioxidant activity can be clearly observed in unprocessed garlic (0 day), $0.314 \pm 0.012$ in DPPH, $0.141 \pm 0.005$ in FRAP, $0.137 \pm 0.001$ in ABTS, respectively. Compared to the lowest value of antioxidant activities in garlic (21 day), the data in unprocessed garlic (0 day) is about 8.7 fold in DPPH, 3.6 fold in FRAP, 2.6 fold in ABTS, in significant level $(p<0.05)$. It was demonstrated that there were great loss of antioxidant activities in "Laba" garlic during traditional processing, and this may be associated with the contents decrease of organosulfur compounds. Interestingly, antioxidant activities (DPPH, ABTS and FRAP) of "Laba" garlic samples remained stable from day 9 to day 15, indicating that the changes of the composition of "Laba" garlic samples during this time have presented little effect on their antioxidant activities.

Correlations between metabolites and antioxidant activities (DPPH, ABTS and FRAP) of "Laba" garlic were created (Table 5) according to the Pearson's correlation coefficients $(r)$ and $p$ values. Six volatile metabolites (diallyl tetrasulphide, 3-vinyl-1,2dithiacyclohex-4-ene, 3,4-dimethylthiophene, sulfur dioxide, trans-3,5-diethyl-1,2,4-trithiolane and propene), two organic acids (DL-malic acid, $n$-octanoic acid) and one amino acid (Ltyrosine) showed a prominent positive correlation with antioxidant activity $(1>r>0.7$ and $p<0.05)$. However, most biological properties of garlic have been reported due to organosulfur compounds. ${ }^{49}$ For instance, Meng et al. ${ }^{50}$ reported a number of organosulfur compounds, which showed positive relationship with the antioxidant capacities of garlic (storing at 8 weeks). Locatelli et al. ${ }^{51}$ also studied organosulfur compounds in the cooked garlic samples, which demonstrated that diallyl sulfide, diallyl disulfide, and diallyl trisulfide showed a lower antioxidant activities, while allicin, ajoenes and vinyldithiins appeared notable antioxidant activities. In our study, diallyl tetrasulphide, 3-vinyl-1,2-dithiacyclohex-4-ene, 3,4-dimethylthiophene, sulfur dioxide and trans-3,5-diethyl-1,2,4-trithiolane showed positive correlations with antioxidant capacities, indicating the strong antioxidant activities of organosulfur compounds. Furthermore, malic acid, tyrosine were previously reported to have antioxidant properties, ${ }^{52,53}$ which was also proved in our study. Phenolic compounds, such as caffeic acid, vanillic acid and sinapic acid, are known as antioxidants. However, four phenolic compounds (caffeic acid, $p$-hydroxybenzoic, vanillic acid and sinapic acid) showed negative correlations with antioxidant activities of "Laba" garlic in our study. This result indicated that phenolic compounds contributed little to the antioxidant activity of "Laba" garlic. Similar pattern was seen in gochujang, the flavonoids of which showed a negative correlation with antioxidant activity. ${ }^{54}$ And this phenomenon is consistent with the previous report of Soto et al. ${ }^{55}$ who has proved that the antioxidant activity of garlic is more associated with organosulfur compounds than phenolic compounds. In addition, organosulfur compounds, organic acids and amino acids showed strong positive correlations with antioxidant activities. Whereas, non-organosulfur compounds, sugars and sugar alcohols and phenolic compounds exhibited negative correlations with antioxidant activities.

Comparing with fresh garlic, the "Laba" garlic at day 12 shows good green colour (absorbances at $590 \mathrm{~nm}$ and $440 \mathrm{~nm}$ were 0.239 and 0.788 respectively), and concentrations of organosulfur compounds decreased by $5.7 \%$, while that of nonorganosulfur compounds increased by $12.8 \%$. The contents of the 15 phenolic compounds reached its maximum value at day 12. Thus, day 12 seemed to be the most suitable time to obtain the "Laba" garlic considering colour, flavor, taste and physiological function. Besides, "Laba" garlic could be stored for more than three months by vacuum packing, light-free and cryopreservation. ${ }^{56}$

Table 4 Antioxidant activities (DPPH, ABTS and FRAP) of garlic samples during processing

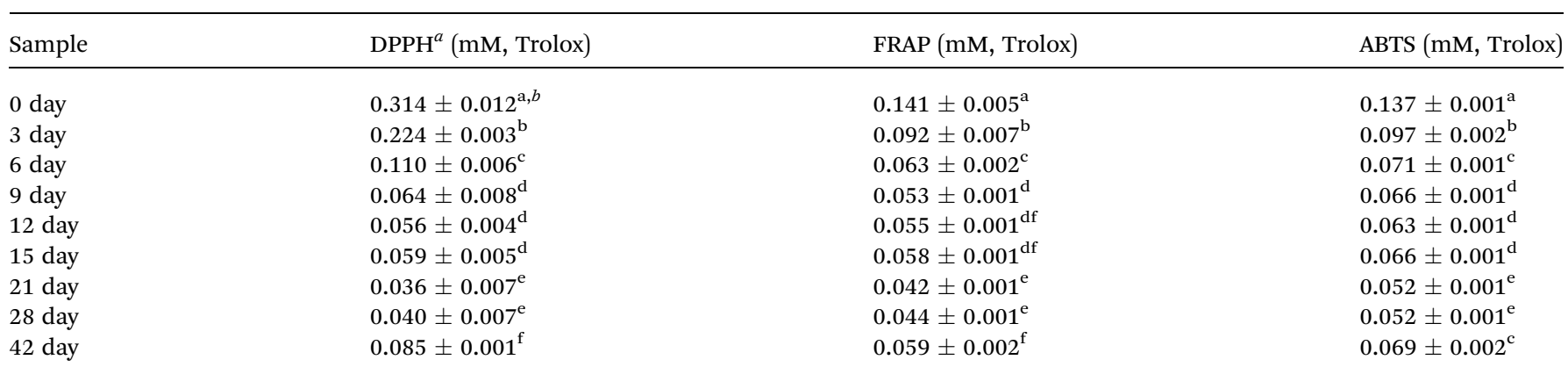

${ }^{a}$ Mean $(n=3) \pm$ standard deviation. ${ }^{b}$ The letters indicate significant differences determined by Duncan's multiple range tests $(p<0.05)$. 
Table 5 Correlations between metabolites and antioxidant activities of "Laba" garlic ${ }^{a}$

\begin{tabular}{|c|c|c|c|c|c|c|c|}
\hline Compound & ABTS & FRAP & DPPH & Compound & ABTS & FRAP & DPPH \\
\hline Allyl methyl sulfide & $-0.78 *$ & $-0.77^{*}$ & $-0.84^{*}$ & Pentitol & $-0.79^{*}$ & $-0.82 *$ & $-0.79 *$ \\
\hline Allyl methyl disulfide & -0.57 & -0.61 & -0.52 & D-Fructose & $-0.9^{*}$ & $-0.91^{*}$ & $-0.86^{*}$ \\
\hline Diallyl sulfide & $-0.75^{*}$ & $-0.74^{*}$ & $-0.77^{*}$ & Sucrose & 0.59 & 0.62 & 0.57 \\
\hline Diallyl disulfide & -0.25 & -0.27 & -0.18 & 1,3-Propanediol & $-0.85^{*}$ & $-0.84^{*}$ & $-0.91 *$ \\
\hline Diallyl trisulfide & $-0.84^{*}$ & $-0.83^{*}$ & $-0.85^{*}$ & Myo-inositol & 0.042 & 0.01 & 0.02 \\
\hline 3,4-Dimethylthiophene & $0.97^{*}$ & $0.97^{*}$ & $0.98^{*}$ & $p$-Coumaric acid & -0.51 & -0.49 & -0.49 \\
\hline 1,3-Dithiole-2-thione & 0.26 & 0.26 & 0.25 & Caffeic acid & $-0.81^{*}$ & $-0.81^{*}$ & $-0.79 *$ \\
\hline Sulfur dioxide & $0.96^{*}$ & $0.96^{*}$ & $0.99^{*}$ & $p$-Hydroxybenzoic & $-0.71 *$ & $-0.71^{*}$ & $-0.76^{*}$ \\
\hline Trans-3,5-Diethyl-1,2,4-trithiolane & $0.98^{*}$ & $0.98^{*}$ & $0.97^{*}$ & Vanillic acid & $-0.72 *$ & $-0.71^{*}$ & $-0.74 *$ \\
\hline Propyl ethynyl sulfoxide & -0.45 & -0.48 & -0.34 & Quercitrin & 0.37 & 0.36 & 0.52 \\
\hline Methyl propenyl disulfide & -0.66 & -0.68 & $-0.71^{*}$ & Ferulic acid & -0.65 & -0.64 & -0.66 \\
\hline Propene & $0.85^{*}$ & $0.87^{*}$ & $0.81^{*}$ & Apigenin & -0.57 & -0.54 & -0.57 \\
\hline Acetaldehyde & 0.21 & 0.19 & 0.06 & Kaempferol & -0.42 & -0.43 & -0.41 \\
\hline Lactic acid & $-0.81^{*}$ & $-0.79^{*}$ & $-0.86^{*}$ & Isorhamnetin & -0.54 & -0.54 & -0.51 \\
\hline DL-Malic acid & $0.83 *$ & $0.85^{*}$ & $0.78^{*}$ & Organosulfur compounds & $0.68^{*}$ & $0.67 *$ & $0.75^{*}$ \\
\hline$n$-Octanoic acid & $0.97^{*}$ & $0.97^{*}$ & $0.98^{*}$ & Non-organosulfur compounds & $-0.68^{*}$ & $-0.67 *$ & $-0.75 *$ \\
\hline Isocitric acid & -0.61 & -0.61 & -0.66 & Organic acids & $0.88^{*}$ & $0.89 *$ & $0.83 *$ \\
\hline L-Alanine & 0.13 & 0.11 & 0.24 & Amino acids & $0.94^{*}$ & $0.93^{*}$ & $0.94^{*}$ \\
\hline L-Leucine & -0.36 & -0.38 & -0.37 & Sugars and sugar alcohols & $-0.93^{*}$ & $-0.91^{*}$ & $-0.92 *$ \\
\hline L-Proline & 0.13 & 0.14 & 0 & Phenolic compounds & $-0.93^{*}$ & $-0.93^{*}$ & $-0.94 *$ \\
\hline Glutamic acid & -0.16 & -0.16 & -0.27 & DPPH & 0.98 & 0.98 & 1 \\
\hline L-Tyrosine & $0.97 *$ & $0.97^{*}$ & $0.98^{*}$ & FRAP & 0.99 & 1 & 0.98 \\
\hline Erythritol & $-0.71 *$ & -0.68 & $-0.78^{*}$ & ABTS & 1 & 1 & \\
\hline
\end{tabular}

$a *$ indicates significance at the 0.05 level (two-tailed test), numbers indicate the Pearson's correlation coefficient values $(r)$.

\section{Conclusions}

In conclusion, the compositions and antioxidant activity of "Laba" garlic during traditional processing were significantly changed. In particular, the colour of garlic samples during traditional processing changed from white to green and then to yellow. Along with the colour variation, the concentration of organosulfur compounds decreased while that of nonorganosulfur compounds increased, which have led to the faint spicy flavor of "Laba" garlic. Primary metabolites including lactic acid, isocitric acid, L-leucine, L-proline, Dfructose, D-glucose and erythritol increased from day 3 to 12 , mainly contributing to the sweet and sour taste of "Laba" garlic. The level of the 15 phenolic compounds reached its maximum value at day 12 , and the antioxidant activities decreased from day 0 to day 28. Moreover, our work also identified notable positive associations between organosulfur compounds, organic acids, amino acids and antioxidant activities. Based on the change of compositions associated with colour, flavor, taste and antioxidant activity in "Laba" garlic, day 12 is the important processing time of "Laba" garlic, and also be recommended as the approximate finish time of the traditional processing.

\section{Conflicts of interest}

The authors declare that they have no conflict of interest.

\section{Acknowledgements}

This work has been carried out with support from the National Key Research and Development Programs of China (2016YFD0400201-8, 2017YFF0211000, 2017YFC1601600).

\section{References}

1 E. Block, The chemistry of garlic and onions, Sci. Am., 1985, 252, 114-119.

2 M. Corzo-Martínez, N. Corzo and M. Villamiel, Biological properties of onions and garlic, Trends Food Sci. Technol., 2007, 18, 609-625.

3 F. Kallel, D. Driss, F. Bouaziz, L. Belghith, S. Zouari-Ellouzi, F. chaari, A. Haddar, S. E. Chaabouniab and R. Ghorbel, Polysaccharide from garlic straw: Extraction, structural data, biological properties and application to beef meat preservation, $R S C A d v$., 2014, 5, 6728-6741.

4 A. Elosta, M. Slevin, K. Rahman and N. Ahmed, Aged garlic has more potent antiglycation and antioxidant properties 
compared to fresh garlic extract in vitro, Sci. Rep., 2017, 7, 39613.

5 P. S. Bisen and M. Emerald, Nutritional and Therapeutic Potential of Garlic and Onion (Allium sp.), Curr. Nutr. Food Sci., 2016, 12, 190-199.

6 M. Molina-Calle, F. Priego-Capote and M. D. L. D. Castro, Headspace-GC-MS volatile profile of black garlic vs. fresh garlic: Evolution along fermentation and behavior under heating, LWT-Food Sci. Technol., 2017, 80, 98-105.

7 Y. S. Queiroz, E. Y. Ishimoto, D. H. M. Bastos, G. R. Sampaio and E. A. F. S. Torres, Garlic (Allium sativum L.) and ready-toeat garlic products: in vitro antioxidant activity, Food Chem., 2009, 115, 371-374.

8 B. Bing, L. Lei, H. X. Song, W. Z. Fu and Z. G. Hua, Increase in the permeability of tonoplast of garlic (Allium sativum) by monocarboxylic acids, J. Agric. Food Chem., 2006, 54, 8103-8107.

9 B. Bing, C. Fang, W. Z. Fu, L. X. Jun, Z. G. Hua and H. X. Song, Mechanism of the greening colour formation of "Laba" garlic, a traditional homemade Chinese food product, $J$. Agric. Food Chem., 2005, 53, 7103-7107.

10 L. Lei, H. Dan, J. Ying, C. Fang, H. X. Song and Z. G. Hua, Relationship between gamma-glutamyl transpeptidase activity and garlic greening, as controlled by temperature, J. Agric. Food Chem., 2008, 56, 941.

11 W. Dan, H. Nanding, H. Na, C. Fang and Z. G. Hua, 2-(1HPyrrolyl) carboxylic acids as pigment precursors in garlic greening, J. Agric. Food Chem., 2008, 56, 1495-1500.

12 T. D. Dan, Z. Bin, Z. L. Yao, H. X. Song, L. X. Jun and Z. Yan, Kinetics of "Laba" garlic greening and its physiochemical properties treated by dense phase carbon dioxide, $L W T$ Food Sci. Technol., 2015, 64, 775-780.

13 S. Imai, K. Akita, M. Tomotake and H. Sawada, Identification of two novel pigment precursors and a reddish-purple pigment involved in the blue-green discolouration of onion and garlic, J. Agric. Food Chem., 2006, 54, 843-847.

14 R. Kubec, M. Hrbácová, R. A. Musah and J. Velísek, Allium discolouration: precursors involved in onion pinking and garlic greening, J. Agric. Food Chem., 2004, 52, 5089-5094.

15 R. Kubec and J. Velísek, Allium discolouration: The colourforming potential of individual thiosulfinates and amino acids: structural requirements for the colour-developing precursors, J. Agric. Food Chem., 2007, 55, 3491-3497.

16 A. E. Pure and M. E. Pure, Antioxidant, antibacterial and colour analysis of garlic fermented in kombucha and red grape vinegar, Appl. Food Biotechnol., 2016, 3, 246-252.

17 S. Y. Lee, S. Lee, S. Lee, J. Y. Oh, E. J. Jeon, H. S. Ryu and C. H. Lee, Primary and secondary metabolite profiling of doenjang, a fermented soybean paste during industrial processing, Food Chem., 2014, 165, 157-166.

18 J. G. Baek, S. M. Shim, D. Y. Kwon, H. K. Choi, C. H. Lee and Y. S. Kim, Metabolite profiling of Cheonggukjang, a fermented soybean paste, during fermentation by gas chromatography-mass spectrometry and principal component analysis, Food Chem., 2010, 122, 1313-1319.

19 D. Chen, Y. Ye, J. Chen and X. Yan, Evolution of metabolomics profile of crab paste during fermentation, Food Chem., 2016, 192, 886-892.
20 A. C. Kimbaris, N. G. Siatis, D. J. Daferera, P. A. Tarantilis, C. S. Pappas and M. G. Polissiou, Comparison of distillation and ultrasound-assisted extraction methods for the isolation of sensitive aroma compounds from garlic (Allium sativum), Ultrason. Sonochem., 2006, 13, 54-60.

21 F. Priego-Capote and M. D. L. D. Castro, HS-GC/MS volatile profile of different varieties of garlic and their behavior under heating, Anal. Bioanal. Chem., 2016, 408, 3843-3852.

22 S. N. Lee, N. S. Kim and D. S. Lee, Comparative study of extraction techniques for determination of garlic flavor components by gas chromatography-mass spectrometry, Anal. Bioanal. Chem., 2003, 377, 749-756.

23 J. Lisec, N. Schauer, J. Kopka, L. Willmitzer and A. R. Fernie, Gas chromatography mass spectrometry-based metabolite profiling in plants, Nat. Protoc., 2006, 1, 387-396.

24 N. Rangkadilok, S. Sitthimonchai, L. Worasuttayangkurn, C. Mahidol, M. Ruchirawat and J. Satayavivad, Evaluation of free radical scavenging and antityrosinase activities of standardized longan fruit extract, Food Chem. Toxicol., 2007, 45, 328-336.

25 R. Re, N. Pellegrini, A. Proteggente, A. Pannala, M. Yang and C. Rice-Evans, Antioxidant activity applying an improved ABTS radical cation decolourization assay, Free Radicals Biol. Med., 1999, 26, 1231-1237.

26 E. Kovats, Gas chromatographic characterization of organic substances in the retention index system, Adv. Chromatogr., 1965, 1, 229-247.

27 R. Kubec, P. Curko, P. Urajová, J. Rubert and J. Hajšlová, Allium Discoloration: Color Compounds Formed during Greening of Processed Garlic, J. Agric. Food Chem., 2017, 65, 10615-10620.

28 P. Dasgupta, A. Bhattacharya, R. Pal, A. K. Dasgupta and S. Sengupt, Synthesis of diallyl disulfide (DADS) induced gold nanoparticles: characterization and study of its biological activity in human leukemic cell-lines, RSC Adv., 2015, 5, 18429-18437.

29 B. Das and K. Chaudhuri, Amelioration of sodium arsenite induced toxicity by diallyl disulfide, a bioactive component of garlic: the involvement of antioxidants and the chelate effect, RSC Adv., 2014, 4, 20964-20973.

30 A. Montaño, F. J. Casado, A. D. Castro, A. H. Sánchez and L. Rejano, Vitamin Content and Amino Acid Composition of Pickled Garlic Processed With and Without Fermentation, J. Agric. Food Chem., 2004, 52, 7324-7330.

31 Y. Ueda, H. Kawajiri, N. Miyamura and R. Miyajima, Content of some sulfur-containing components and free amino acids in various strains of garlic, J. Jpn. Soc. Food Sci., 2009, 38, 429-434.

32 P. M. Chandrashekara and Y. P. Venkatesh, Immunostimulatory properties of fructans derived from raw garlic (Allium sativum L.), Bioact. Carbohydr. Diet. Fibre, 2016, 8, 65-70.

33 P. M. Chandrashekar, K. V. H. Prashanth and Y. P. Venkatesh, Isolation, structural elucidation and immunomodulatory activity of fructans from aged garlic extract, Phytochemistry, 2011, 72, 255-264. 
34 A. T. Mankarios, C. F. G. Jones, M. C. Jarvis, D. R. Threfall and J. Friend, Hydrolysis of plant polysaccharides and GLC analysis of their constituent neutral sugars, Phytochemistry, 1979, 18, 419-422.

35 V. Lanzotti, Bioactive polar natural compounds from garlic and onions, Phytochem. Rev., 2012, 11, 179-196.

36 G. Oboh, A. J. Akinyemi and A. O. Ademiluyi, Inhibitory effect of phenolic extract from garlic on angiotensin-1 converting enzyme and cisplatin induced lipid peroxidation-in vitro, Int. J. Biomed. Sci., 2013, 9, 98-106.

37 V. M. Beato, F. Orgaz, F. Mansilla and A. Montaño, Changes in phenolic compounds in garlic (Allium sativum L.) owing to the cultivar and location of growth, Plant Foods Hum. Nutr., 2011, 66, 218-223.

38 M. I. Alarcón-Flores, R. Romero-González, J. L. M. Vidal and A. G. Frenich, Determination of Phenolic Compounds in Artichoke, Garlic and Spinach by Ultra-High-Performance Liquid Chromatography Coupled to Tandem Mass Spectrometry, Food Anal. Methods, 2014, 7, 2095-2106.

39 F. Fratianni, M. N. Ombra, A. Cozzolino, R. Riccardi, P. Spigno, P. Tremonte, R. Coppola and F. Nazzaro, Phenolic constituents, antioxidant, antimicrobial and antiproliferative activities of different endemic Italian varieties of garlic (Allium sativum L.), J. Funct. Foods, 2016, 21, 240248.

40 M. I. Torino, R. I. Limón, C. Martínez-Villaluenga, S. Mäkinen, A. Pihlanto, C. Vidal-Valverde and F. Juana, Antioxidant and antihypertensive properties of liquid and solid state fermented lentils, Food Chem., 2013, 136, 10301037.

41 C. M. Ajila, S. K. Brar, M. Erma, R. D. Tyagi and J. R. Valéro, Solid-state fermentation of apple pomace using Phanerocheate chrysosporium-liberation and extraction of phenolic antioxidants, Food Chem., 2011, 126, 1071-1080.

42 B. Verma, P. Hucl and R. N. Chibbar, Phenolic acid composition and antioxidant capacity of acid and alkali hydrolysed wheat bran fractions, Food Chem., 2009, 116, 947-954.

43 S. Mathew and T. E. Abraham, Ferulic acid: an antioxidant found naturally in plant cell walls and feruloyl esterases involved in its release and their applications, Crit. Rev. Biotechnol., 2004, 24, 59-83.

44 M. G. Hertog, E. J. Feskens, P. C. Hollman, M. B. Katan and D. Kromhout, Dietary antioxidant flavonoids and risk of coronary heart disease: the Zutphen Elderly study, Lancet, 1993, 342, 1007-1011.
45 P. A. Tuan, N. I. Park and X. Li, Molecular Cloning and Characterization of Phenylalanine Ammonia-lyase and Cinnamate 4-Hydroxylase in the Phenylpropanoid Biosynthesis Pathway in Garlic (Allium sativum), J. Agric. Food Chem., 2010, 58, 12176.

46 H. Chenjiang and C. T. Ho, Antioxidant Activities of Caffeic Acid and Its Related Hydroxycinnamic Acid Compounds, J. Agric. Food Chem., 1997, 45(7), 2374-2378.

47 M. Nardini, M. D'Aquino, G. Tomassi, V. Gentili, M. Di Felice and C. Scaccini, Inhibition of human low-density lipoprotein oxidation by caffeic acid and other hydrocinnamic acid derivatives, Free Radical Biol. Med., 1995, 19, 541-552.

48 W. Q. Li, H. Zhou, M. Y. Zhou, X. P. Hu, S. Y. Ou, R. A. Yan, X. J. Liao, X. S. Huang and L. Fu, Characterization of Phenolic Constituents Inhibiting the Formation of SulfurContaining Volatiles Produced During Garlic Processing, $J$. Agric. Food Chem., 2015, 63, 787-794.

49 V. Lanzotti, The analysis of onion and garlic, J. Chromatogr. A, 2006, 1112, 3-22.

50 L. F. Meng, L. Tong, L. Wei and Y. L. De, Changes in antioxidant capacity, levels of soluble sugar, total polyphenol, organosulfur compound and constituents in garlic clove during storage, Ind. Crops Prod., 2015, 69, 137142.

51 D. Locatelli, M. Nazareno, F. Cecilia and C. Alejandra, Cooked garlic and antioxidant activity. Correlation with organosulphur compound compositions, Food Chem., 2017, 220, 219-224.

52 K. Papadopoulos, T. Triantis, D. Dimotikali and J. Nikokavouras, Evaluation of food antioxidant activity by photostorage chemiluminescence, Anal. Chim. Acta, 2001, 433, 263-268.

53 S. J. Hur, S. Y. Lee, Y. C. Kim, I. Choi and G. B. Kim, Effect of fermentation on the antioxidant activity in plant-based foods, Food Chem., 2014, 160, 346-356.

54 E. L. Da, G. R. Shin, S. Lee, E. S. Jang, H. W. Shin, B. S. Moon and $\mathrm{C}$. H. Lee, Metabolomics reveal that amino acids are the main contributors to antioxidant activity in wheat and rice gochujangs (Korean fermented red pepper paste), Food Res. Int., 2016, 87, 10.

55 C. Soto, R. González, M. M. Sance and C. Galmarini, Organosulfur and phenolic content of garlic (Allium sativum L.) and onion (Allium cepa L.) and its relationship with antioxidant activity, Acta Hortic., 2016, 39, 277-290.

56 B. Bing, J. S. Juan, W. D. Mei, L. Y. Ping and J. D. Hua, Methods and influence factors in green protecting of "Laba" garlic, Sci. Technol. Food Ind., 2011, 32, 129-132. 